\title{
A review of paediatric abdominal
}

\section{masses}

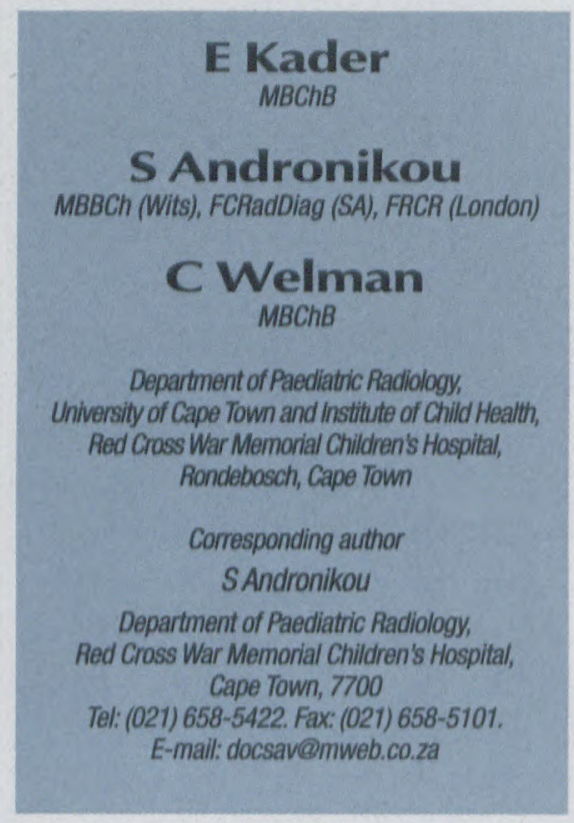

\section{Abstract}

The extensive differential diagnosis of a paediatric abdominal mass may be considerably narrowed by considering the clinical information and radiographic characteristics. Less than half of abdominal masses are surgical and, of these, nearly all are retroperitoneal, and usually renal. A neonatal abdominal mass is usually benign, retroperitoneal and renal, caused in particular by hydronephrosis and multicystic dysplastic kidney. The commonest neonatal benign and malignant tumours are mesoblastic nephroma and sacrococcygeal teratoma respectively. In the older child abdominal masses are most likely to be malignant retroperitoneal tumours, especially neuroblastoma and Wilm's tumour. The commonest benign mass in this age group is appendix abscess. The initial radiological investigation is a plain abdominal film. In the neonate, where the most likely cause is a benign cystic mass, ultrasound is the preferred subsequent investigation. In the older child, where the risk of malignancy is higher, it may be justifiable to use CT in preference to ultrasound. The spectrum of abdominal masses in paediatric practice is diverse, but a knowledge of the statistical distribution, clinical features and imaging characteristics allows the formulation of a focused differential.

\section{Key words}

Hydronephrosis, teratoma, neuroblastoma, Wilm's appendix mass

\section{Introduction}

While the differential of an abdominal mass in the paediatric age group is diverse, it is worth noting that only a few conditions account for the vast majority of cases. They occur at any age and have a broad spectrum of clinical presentations. A knowledge of the statistical distribution of paediatric abdominal masses, the age and sex of the patient and a guided clinical evaluation as well as the radiological imaging characteristics allow the formulation of a focused differential diagnosis, and in some cases a specific diagnosis. 


\section{from page 14}

\section{Diagnostic imaging}

Fifty-seven per cent of abdominal masses are non-surgical and caused by organomegaly related to medical conditions (Figure 1), while $43 \%$ are sur-

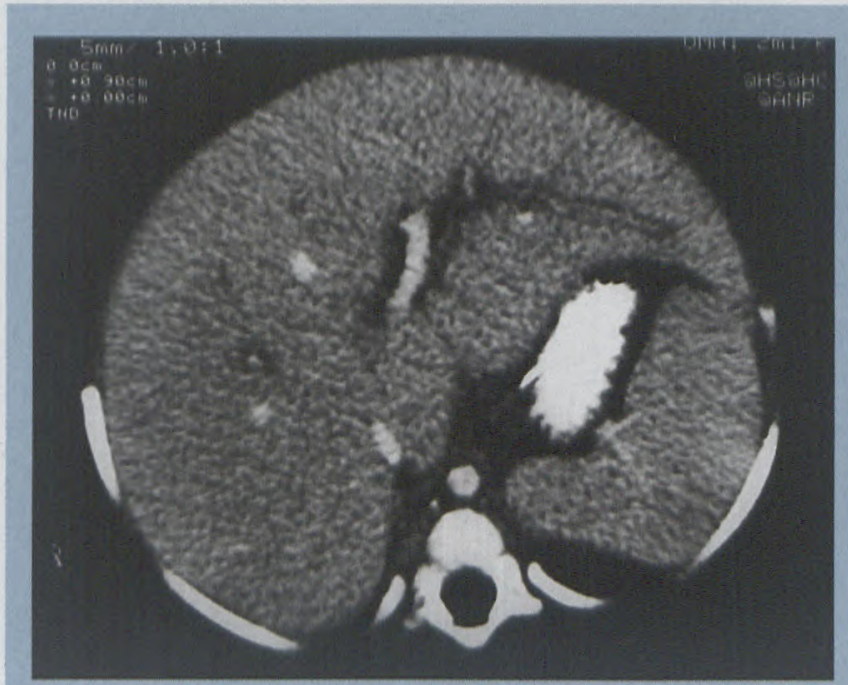

Figure 1: Homogeneous hepatomegaly in a patient with tuberculosis

over $50 \%$ of cases. ${ }^{2,3}$ The $13 \%$ accounting for malignant disease includes sacrococcygeal teratoma, the commonest new-born tumour, as well as neuroblastoma, Wilm's tumour and hepatoblastoma ${ }^{1 / 4}$ (see Table 2).

The most $\mathrm{com} \mathrm{mon}$ causes of neonatal masses are hydronephrosis and multicystic dysplastic kidney, which account for $40 \%$ of neonatal abdominal masses. ${ }^{2,3,5}$ Less common renal causes include renal vein thrombosis, gical in origin. ${ }^{1}$ Some $80-90 \%$ of surgical abdominal masses are retroperitoneal, two-thirds of which are renal and one third extra-renal (and usually malignant). ${ }^{1,2}$ Only $10-20 \%$ are intra-peritoneal (see Table 1).

In the neonate, an abdominal mass is most likely benign (87\%), retroperitoneal $(65 \%)$ and of urological origin in

\section{Table 1: Causes of paediatric abdominal masses according to site of pathology}

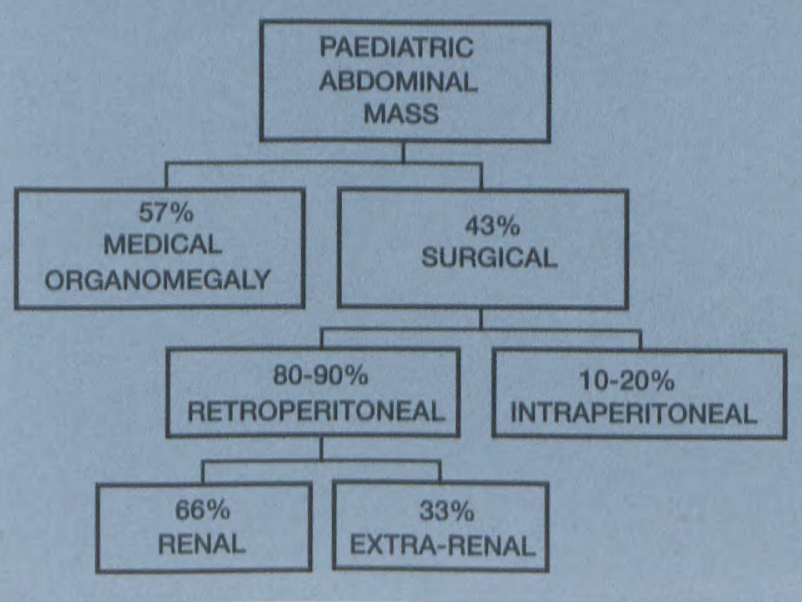
polycystic kidney disease and mesoblastic nephroma (the most common neonatal intrarenal solid mass and second only to sacrococcygeal teratoma in frequency in the new-born). ${ }^{6,7}$ Gastrointestinal lesions account for 15\% of cases and include, most commonly, duplications, segmental bowel dilations, meconium ileus complications and mesenteric/omental cysts. ${ }^{2}$ Genital masses account for $15 \%$ of cases and include hydrometrocolpos and ovarian masses such as cysts and teratomas. ${ }^{2}$ Ten per cent are nonrenal and retroperitoneal and include adrenal haemorrhage, neuroblastoma and teratomas. ${ }^{2}$ Hepatobiliary

\section{Table 2: Most common causes of a neonatal abdominal mass}
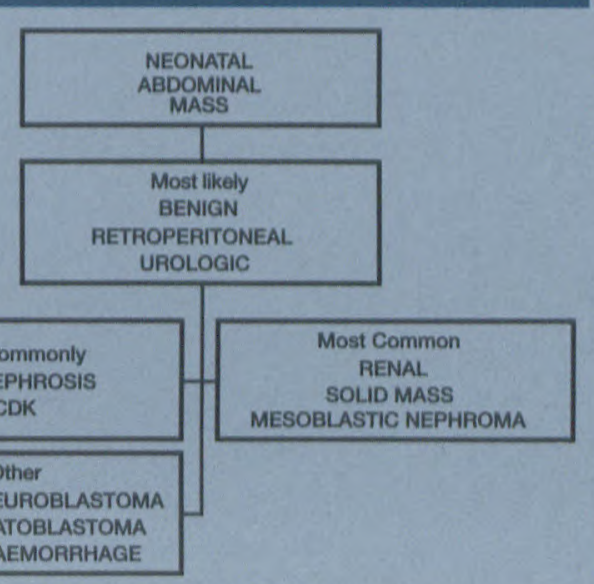

masses account for $5 \%$ of masses and include metastases, haemangioendothelioma, hepatoblastoma and hydrops of the gall bladder. ${ }^{3}$

Abdominal masses are more likely to be malignant in the older child than in the neonate. Retroperitoneal malignancies account for $50-78 \%$ of abdominal masses, reflecting the dramatic increases in non-renal masses, primarily neuroblastoma. ${ }^{1.8}$ Neuroblastoma and hepatoblastoma are more common under two years of age, whereas nephroblastoma (Wilm's tumour), hepatoma, genitourinary rhabdomyosarcoma and ovarian germ cell tumours are more common in older children $^{4}$ (see Table 3). Wilm's tumours

\section{Table 3: Most common causes for an abdominal mass in an older child}

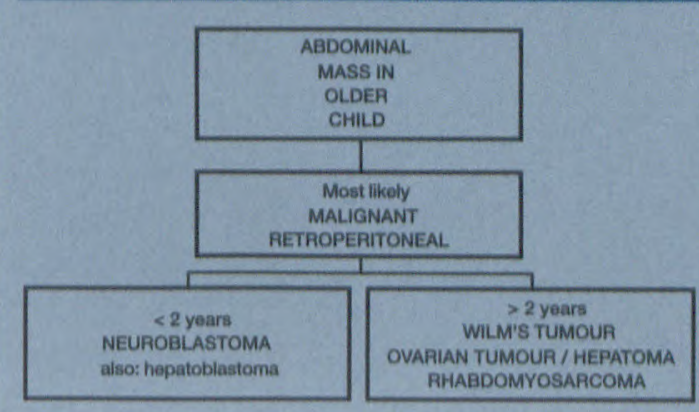




\section{from page 15}

and neuroblastoma account for around $20 \%$ of all malignancies in children one to 15 years of age. Wilm's tumour represents $22 \%$ of all abdominal masses and $7-10 \%$ of malignant tumours in childhood. Neuroblastoma occurs with a similar frequency to Wilm's tumour, representing $21 \%$ of abdominal masses in childhood and $8-10 \%$ of all childhood cancers. $4,7,8$ The most common benign abdominal mass in this age group is an appendix abscess (see Table 4). According to Hilton and

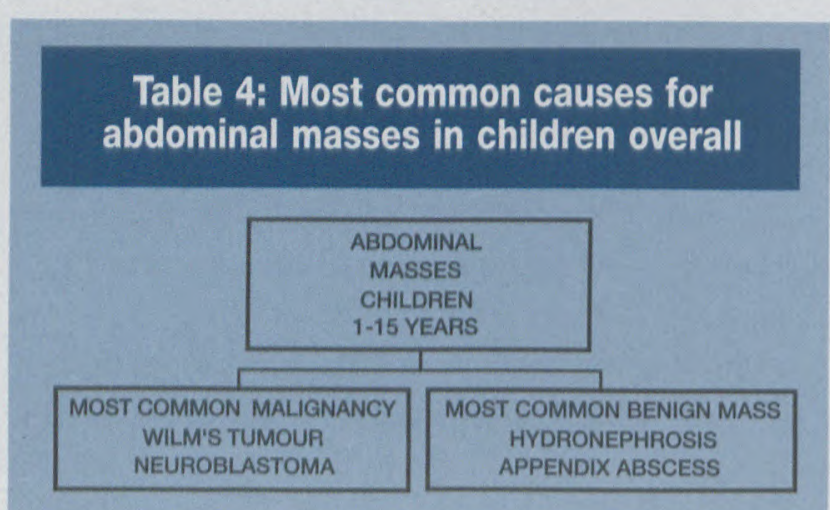

Edwards, the distribution of abdominal masses by organ system is as follows: renal 55\% [Wilm's tumour (22\%), hydronephrosis (20\%)]; non-renal retroperitoneal 23\% [neuroblastoma (21\%), rhabdomyosarcoma, teratoma]; GIT and hepatobiliary 18\% [appendix mass (9-10\%), intussusception (Figure 2), duplication cyst]; and genital $4 \%$ [ovarian masses, hydrometrocolpos]. ${ }^{2,8}$

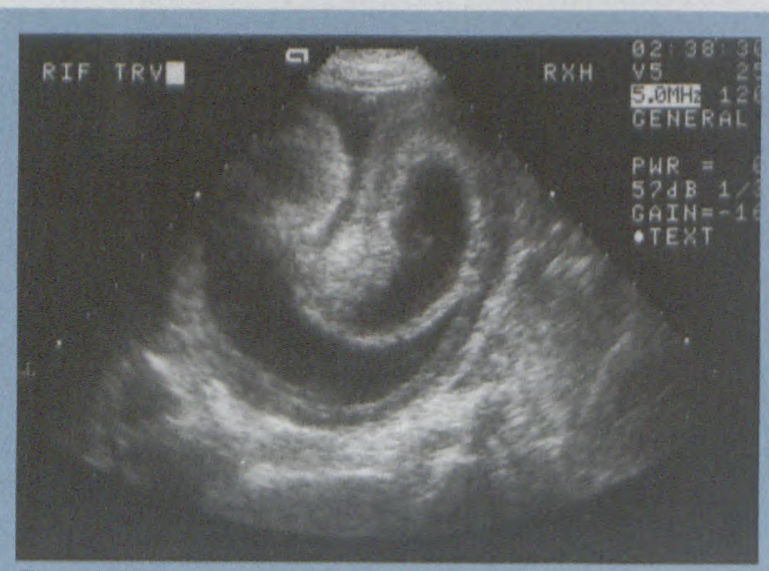

Figure 2: Ultrasound scan of an ileo-caecal intussusception
In the South African setting, infective masses are common in childhood, particularly tuberculosis, which must be included in the differential diagnosis (Figures 3 and 4). Its true incidence is unknown and differentiation from an appendix abscess or lymphoma may be difficult without biopsy. Other common infections and infestations that may simulate masses include renal abscess (Figure 5), appendix mass, hepatic abscess (Figure 6), hydatid cyst and worm bolus (Figure 7).

Following a focused clinical evaluation, the initial radiological investigation should be an abdominal radiograph. The bowel gas pattern is assessed for obstruction or soft tissue masses. If there is evidence of bowel obstruction, the mass is invariably of gastrointestinal origin, as extra-enteric masses do not usually cause obstruction. ${ }^{2}$ Calcification sug-

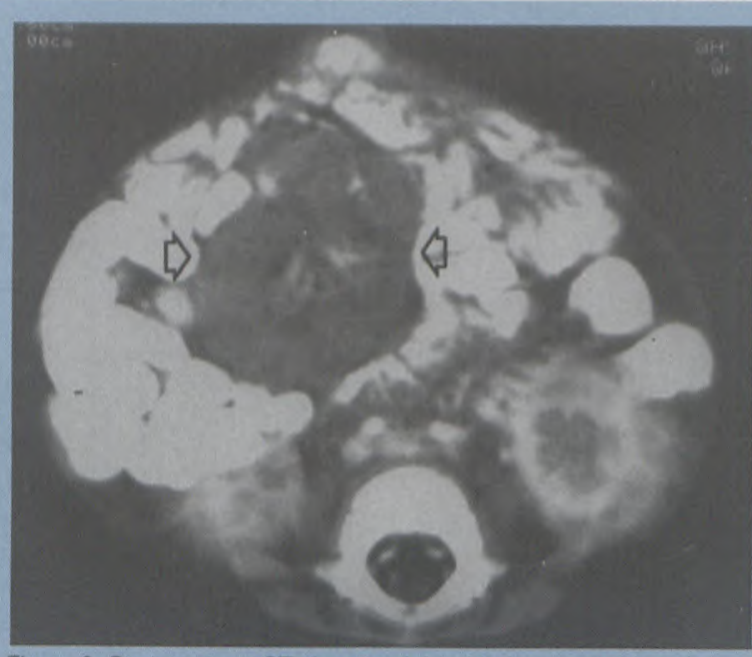

Figure 3: Post-contrast CT shows a right-sided mesenteric mass due to matted tuberculous lymphadenopathy (arrows). Bilateral perinephric stranding is also present.

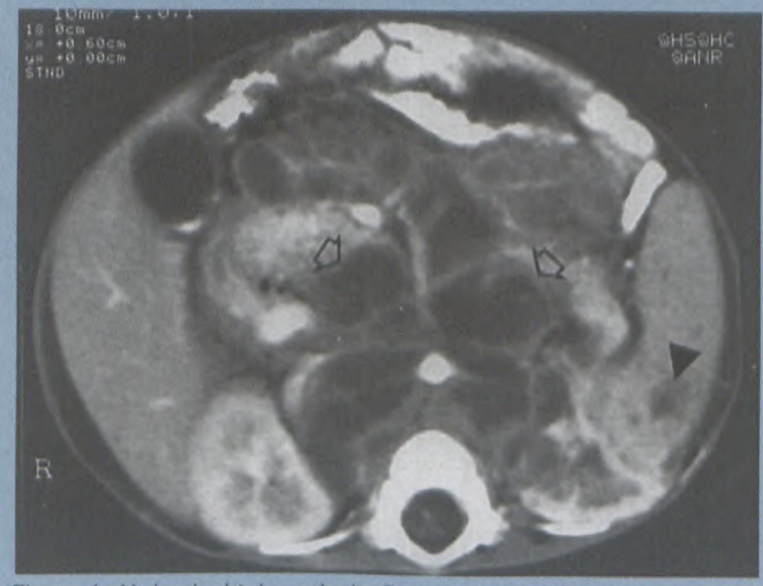

Figure 4: Abdominal tuberculosis: Post-contrast CT shows hypodense rim-enhancing lymph nodes (arrows) splaying the mesenteric vessels. A splenic granuloma is also present (arrowhead).

tion without or with a prior contrast study is indicated. ${ }^{7}$ In the presence of gests neuroblastoma, hepatoblastoma or meconium peritonitis (see Table 5). Bony abnormalities such as sacral deformity may suggest a teratoma and erosion may allude to a neuroblastoma. Where there is obstruction, surgical explora-

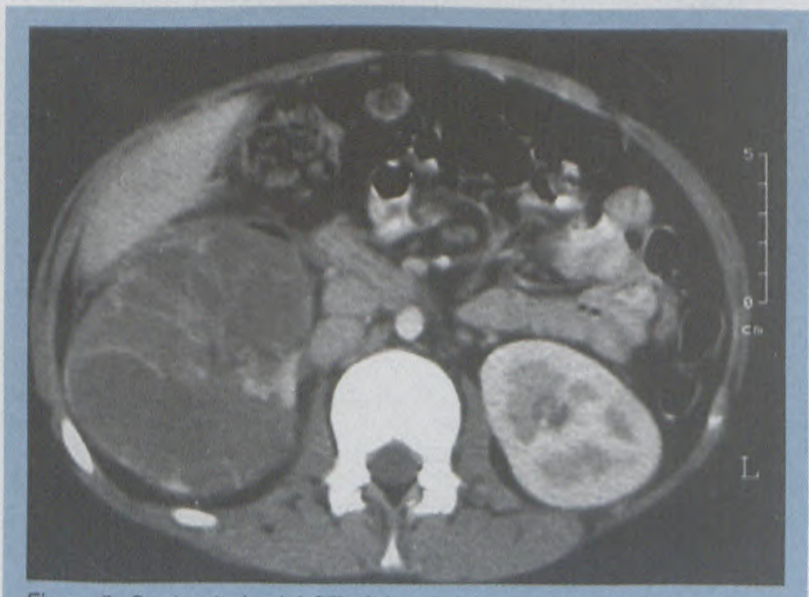

Figure 5: Contrasted axial CT of the abdomen demonstrates poor enhancement of an enlarged, hypodense right kidney which progressed to form an abscess 


\section{A review of paediatric abdominal masses}

from page 16

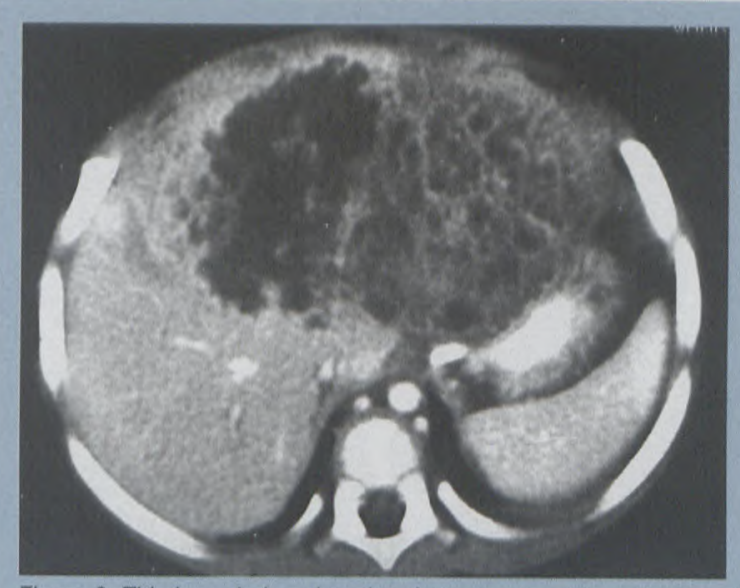

Figure 6: This irregularly-enhancing, heterogeneous hepatic mass was proved to be an abscess

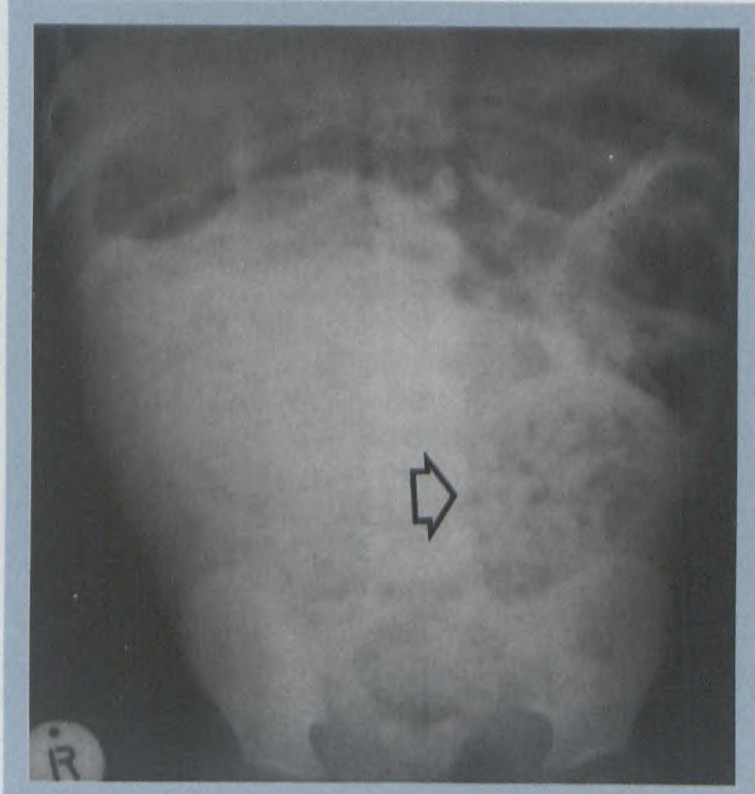

Figure 7: Abdominal X-ray showing a left lower quadrant worm bolus (arrow)

a normal abdominal film, the next appropriate investigation is an ultrasound. ${ }^{3}$ This is frequently diagnostic by virtue of its ability to differentiate solid from cystic masses and to define the organ of origin. Sonography also does not expose the child to ionising radiation, allows guided biopsy of the lesion and assessment of the inferior vena cava for thrombosis - particularly with Wilm's tumour, renal vein thrombosis and liver tumours. Intravenous urography has been supplanted by ultrasound, CT scanning and nuclear scintigraphy.

CT offers superb demonstration of anatomy and allows detection of the organ of origin, assessment of the degree of organ involvement, local and nodal spread, vascular invasion and $\mathrm{m}$ e $\mathrm{t}$ a s $\mathrm{t}$ a $\mathrm{t}$ ic Table 5: Causes for calcification seen on conventional abdominal radiograph

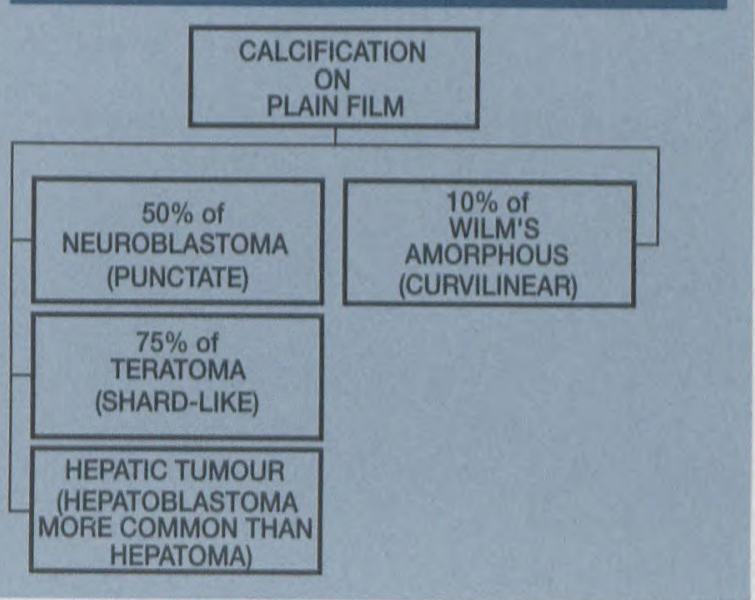

spread. Its position in the investigation protocol should be tailored to the clinical scenario. In the new-born, where the most likely aetiology is a benign cystic lesion, ultrasound is preferred, while in an older child where the probability of malignancy is higher, CT may be the initial investigation.

MRI is an emerging modality with the advantages of multiplanar imaging, vascular assessment without contrast and detection of biochemical differences in tissue. Supplementary investigations such as nuclear medicine scanning, voiding cystourethrography, angiography and venography have a limited role in the assessment of patients with abdominal masses, but add valuable information regarding renal function, urine outflow obstruction and, in the case of neuroblastoma, bony metastatic spread.
We have included a selection of cases that we have encountered with short descriptions of their diagnostic features.

- Multicystic dysplastic kidney (MCDK) (Figure 8) is one of the two most common causes of a neonatal abdominal mass and is the most

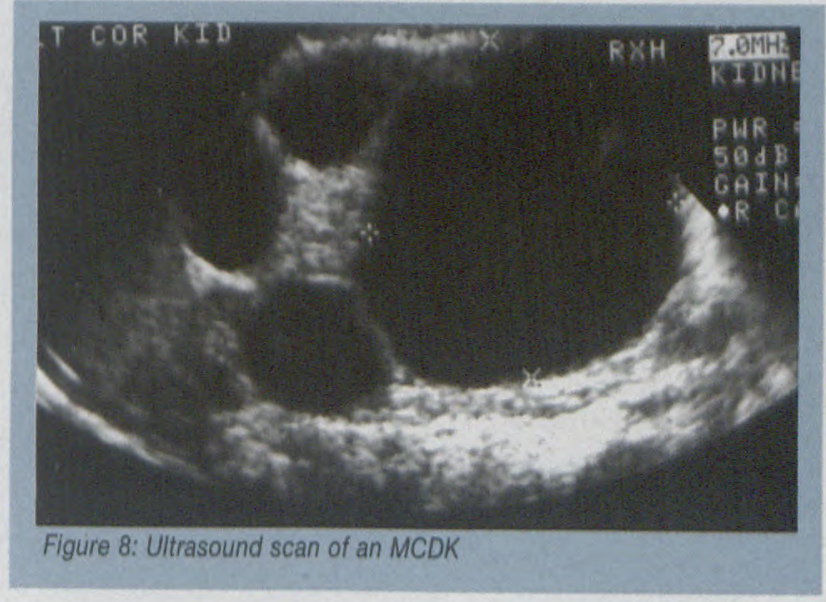

common presenting abdominal mass in the first day of life. The basic developmental defect is ureteropelvic atresia. It is usually asymptomatic, unilateral and situated on the left in $80 \%$ of cases. A third of cases have contralateral renal abnormalities. Ultrasound shows multiple noncommunicating cysts in a "grapecluster" pattern. 


\section{A review of paediatric abdominal masses}

\section{from page 17}

- Hydronephrosis (Figure 9) is the second of the two most common causes of a neonatal abdominal mass and is most commonly due to pelviureteric junction (PUJ)

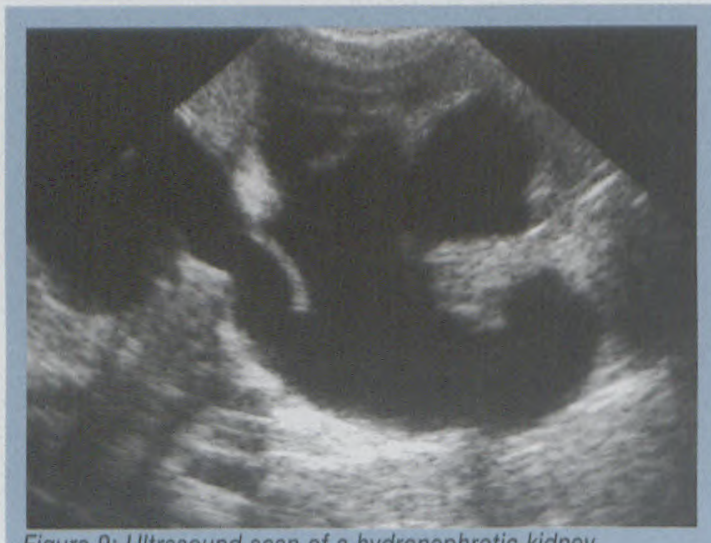

Figure 9: Ultrasound scan of a hydronephrotic kidney.

highly malignant tumour is the third most common palpable abdominal mass in childhood. ${ }^{1}$ It arises anywhere along the sympathetic chain. Some 60$80 \%$ are abdominal - approximately $40-50 \%$ are adrenal and $20-30 \%$ occur in pelvic or visceral ganglia, paraganglia or the organ of Zuckerkandl. ${ }^{4,6,8}$ Some 95\% present before eight years of age (peak incidence is at five years). obstruction $(22 \%)$, posterior urethral valves (PUV) and vesico-ureteric reflux (VUR). ${ }^{3}$ Radionuclide scanning is valuable in assessing renal function and differentiating MCDK from hydronephrosis - which may be difficult to do with ultrasound.

- Renal vein thrombosis. The most common cause in the neonate is dehydration, while in the older child trauma and malignancy are more important causes. Ultrasound shows an enlarged, echogenic kidney and inferior caval venography may confirm the diagnosis.

\section{- Neuroblastoma (Figure 10). This}

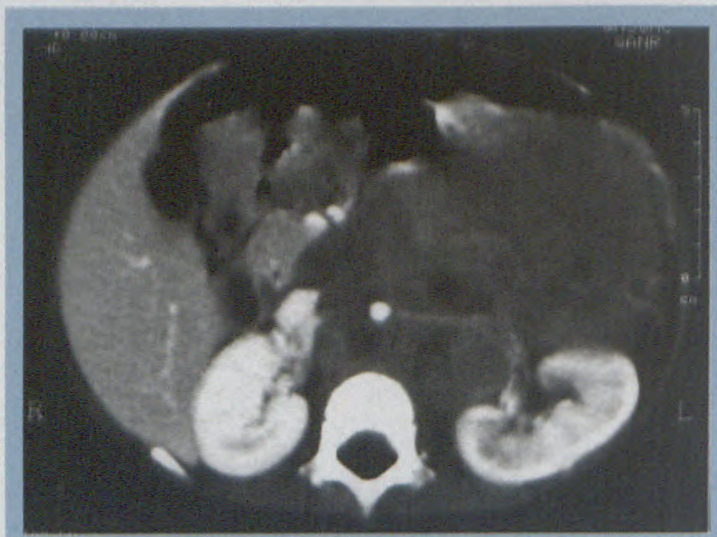

Figure 10: Contrasted abdominal CT. There is an irregular mass anterior to the left kidney, which crosses the midline and encases the aorta (arrow). The features are typical of a neuroblastoma.
Seventy per cent have metastases at presentation. Ultrasound shows a hyperechoic mass with areas of cystic and necrotic change (often seen as a well-defined echogenic lobule associated with a larger tumour mass). ${ }^{2}$

A CT scan demonstrates the tumour's tendency to infiltrate adjacent structures and encase vessels as it crosses the midline, and has a greater sensitivity for detection of calcification compared with plain film. Punctate calcification is present in $50 \%$ of cases. Spontaneous regression into a more benign variant such as ganglioneuroblastoma and ganglioneuroma, more commonly seen in older children, may occur. Prognosis varies inversely with age.

- Wilm's tumour (Figure 11). This is the fourth most common palpable abdominal mass in childhood. ${ }^{1}$ Some $75 \%$ present before five years of age (peak incidence is at three years) and, in contrast to neuroblastoma, only $25 \%$ have metastases at presentation. Ultrasound shows a hyperechoic mass with areas of necrosis and cystic change. CT demonstrates a hypodense, inhomogeneously enhancing mass that is more often left-sided and upper pole in location. Amorphous and curvilinear calcification is present in $10 \%$ and $5-10 \%$ may be bilateral. Fifty per cent cross the midline. The "claw" sign is a valuable indicator that the mass arises from the kidney and represents a splayed, relatively normal portion of parenchyma partially surrounding the mass. Nephroblastomatosis is a rare precursor of Wilm's tumour. It is echogenic, solid and sub-cortical on sonar and is better detected by MRI. - Mesoblastic nephroma (MN) (Figure 12). A solid renal mass in a neonate is most likely an MN. This is a mesenchymal tumour that is part of a spectrum, from a classic benign type, through a cellular type to malignant spindle cell sarcoma. It is usually unilateral. Ultrasound shows a solid, hypoechoic mass with a characteristic "ring sign" of concentric hyper/hypoechoic rings.

- Polycystic kidney disease (PCKD). Infantile PCKD (Figure 13) is 
from page 18

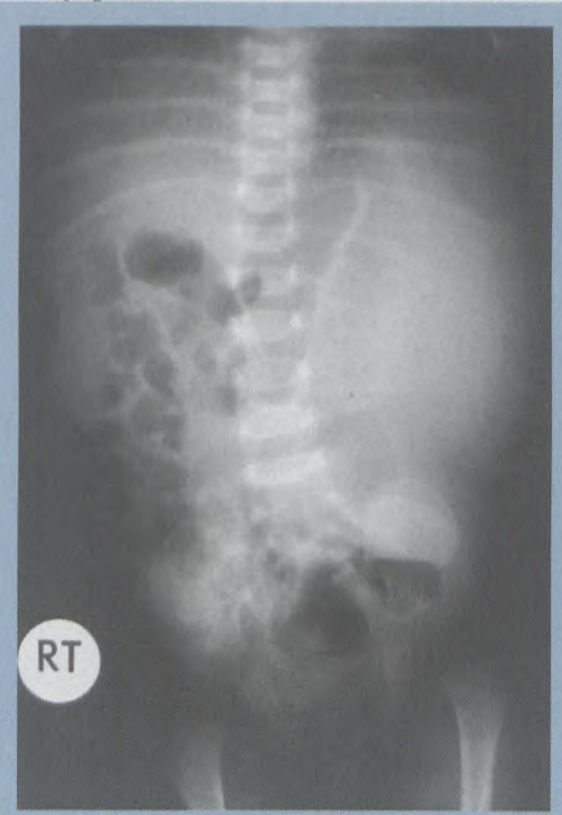

Figure 12: Plain abdominal radiograph showing a large left-sided soft tissue mass displacing the viscera. This was a mesoblastic nephroma.

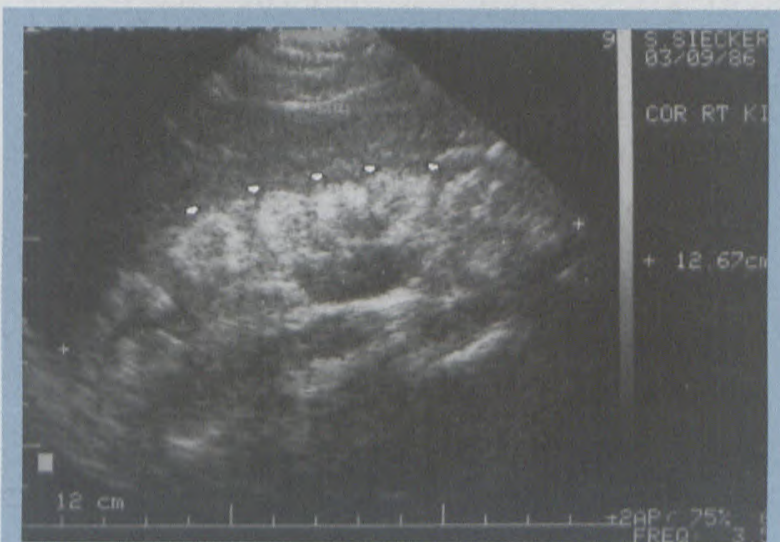

Figure 13: Coronal ultrasound scan showing an enlarged, echogenic right kidney due to infantile polycystic kidney disease (arrows)

characterised by microcysts within the kidneys producing bilateral enlarged, echogenic kidneys on ultrasound. There is associated hepatic fibrosis. The renal and hepatic changes vary by inverse proportion. Adult PCKD is rare at this age and is characterised by multiple hypoechoic cysts. There are associated cysts in other organs, but no hepatic fibrosis.

Other renal masses include infective masses (as mentioned earlier) and infiltrations, including leukaemia (Figure 14) and lymphoma.

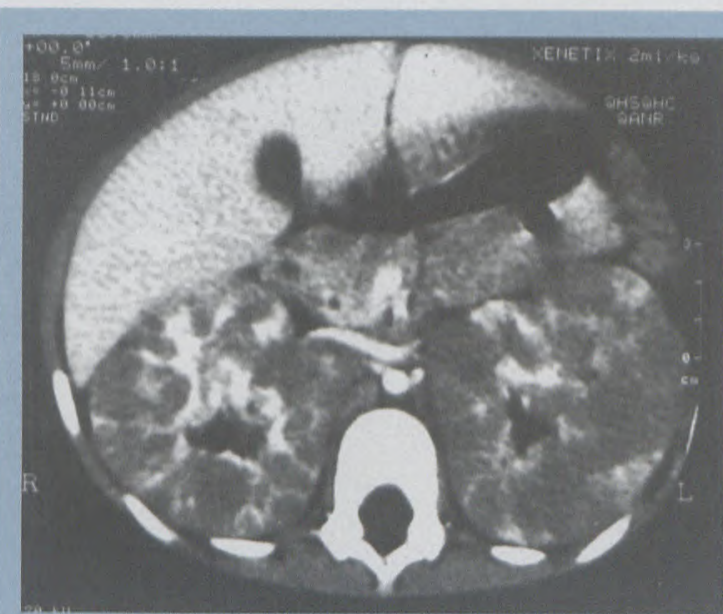

Figure 14: Contrasted abdominal CT. Both kidneys are enlarged and hypodense due to leukaemic infiltration and show enhancement of the compressed normal tissue.

- GIT duplication and enterogenous cysts (Figure 15). These can occur anywhere from mouth to anus,
but are most
common in the
terminal ileal
region and
represent the
most common
enteric cause of
$\mathrm{n}$ e o n a t a 1
a bdom in a 1
masses.

- Omental, mesenteric (cystic lymphangioma) and retroperitoneal cysts (Figure 16). These are believed to be due to lymphatic obstruction. Mesenteric cysts are more common than omental cysts and occur in an older population.

\section{- Primary liver tumours.} Hepatobiliary masses represent approximately $6 \%$ of all paediatric tumours beyond the new-born period. ${ }^{8}$ Metastases from Wilm's tumour, neuroblastoma and lymphoma are more common than primary tumours. Two thirds of primary liver tumours are malignant and a third are benign. Plain film may show calcification, ultrasound will differentiate solid from cystic lesions, while triplephase CT demonstrates anatomy, aids in differentiating lesion types and improves detection of metastases. Angiography, which elucidates the vascular supply, is essential prior to resection or for embolisation.

Common

tumours include haemangiomas (which together with haemangio-

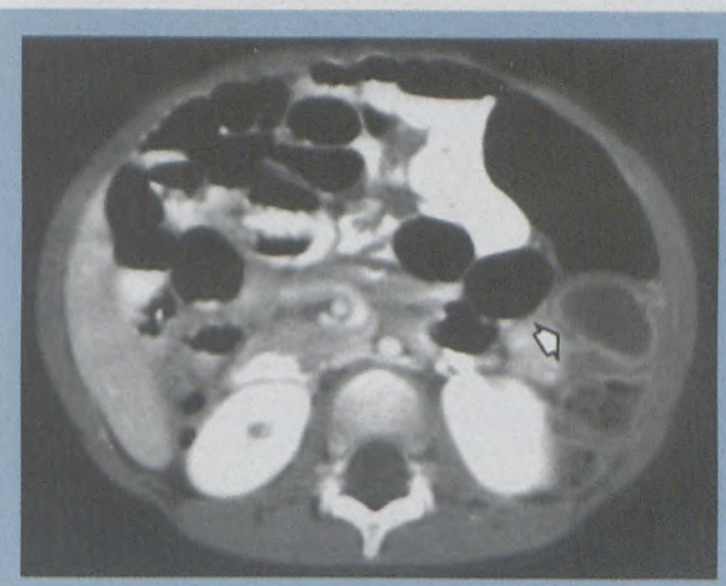

Figure 15. Post-contrast $\mathrm{CT}$ of the abdomen showing an nhancing cyst in the left flank (arrow). This was an enteric cyst.

endothelioma form the most common group of primary liver tumours), hamartomas and adenomas. Haemangioendotheliomas are often multicentric and malignant in 33\% of cases. Hepatoblastomas outnumber hepatomas by $4: 1$. Hepatoblastoma (Figure 17) is more common than hepatocellular carcinoma (HCC) in the under-3 age group and shows a female preponderance. Calcification is present in $10-50 \%$ and is coarse and dense. Hepatoma is more common 


\section{A review of paediatric abdominal masses}

\section{from page 19}

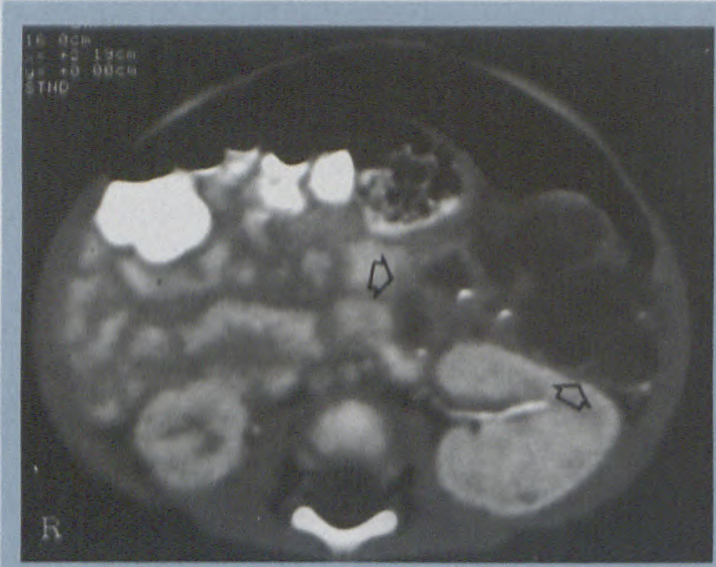

Figure 16. Axial contrasted CT shows an intra-abdominal multicystic mass (arrows) with focal calcification. This proved to be a lymphangioma.

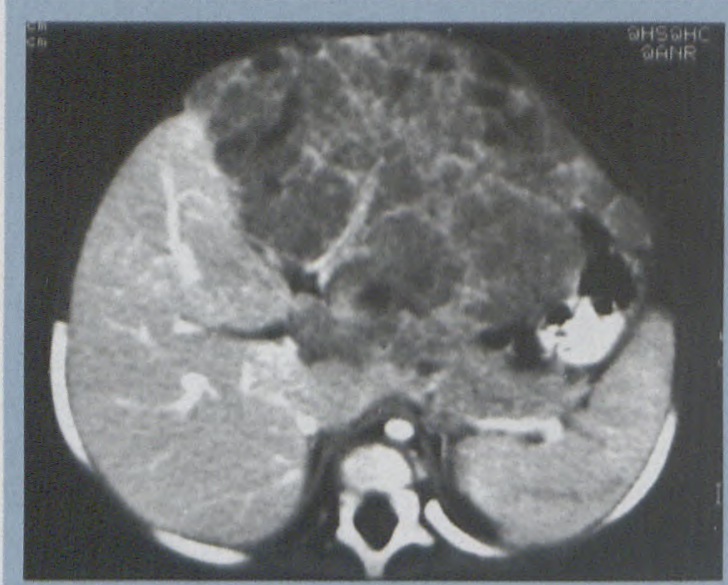

Figure 17: Post-contrast CT of the liver. The inhomogeneous, poorly-enhancing mass in the left hepatic lobe was a hepatoblastoma.

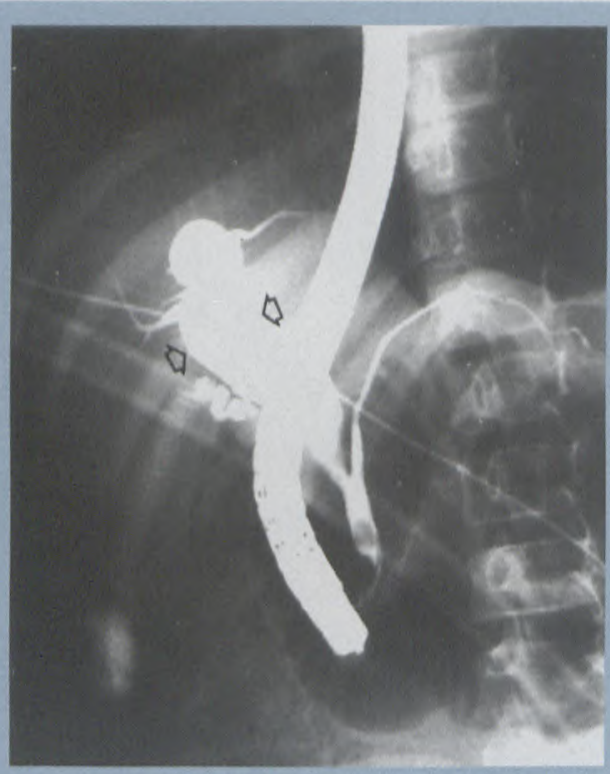

Figure 18: An ERCP demonstrating a choledochal cyst (arrows).

- Ovarian masses. Cystic lesions (Figure 19) are usually benign and include follicles, cystadenomas and dermoids/ teratomas (Figure 20) (the most common ovarian neoplasm in childhood). ${ }^{8}$ The latter tumour shows plain film calcification in $75 \%$ of cases (vide infra). Solid lesions should suggest malignancy. These account for $15-20 \%$ of ovarian masses and are of germ cell origin in $75-80 \%$ of cases. The most common tumour angiography (PTC) and CT. is the malignant teratoma, There is a $3 \%$ risk of followed by dysgerminoma. malignancy.

- Pelvic masses. In the female the mass is usually of uterine or ovarian origin (vide infra). In the male, a distended bladder secondary to posterior urethral valves is the

in the 4-5 year age group and shows a male preponderance. Calcification is present in $10-25 \%$.

- Choledochal cyst (Figure 18). These are believed to arise secondary to reflux of pancreatic secretions into the biliary tree. Five types are recognised and classified. Sixty per cent occur under 10 years of age. There is a female to male ratio of $4: 1$. Useful investigations include ultrasound, endoscopic retrograde pancreatography (ERCP), percutaneous transhepatic chol-

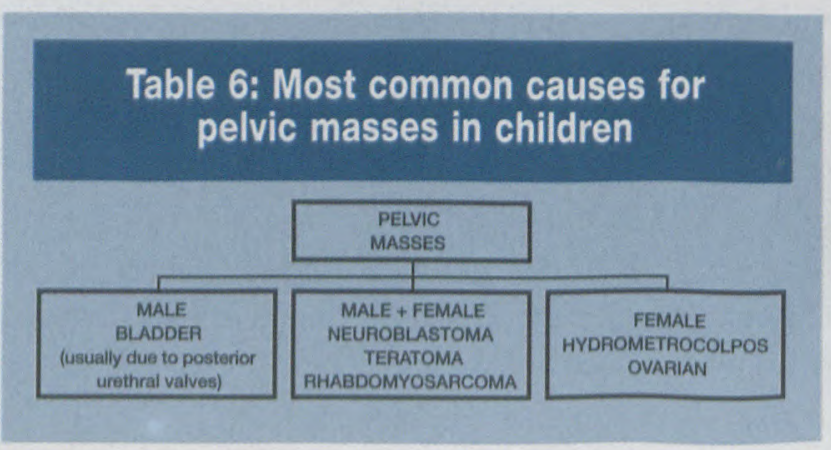

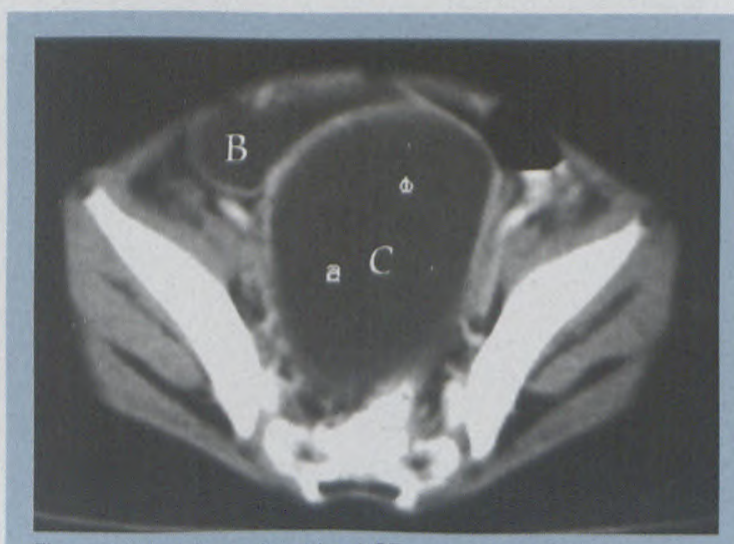

Figure 19: Pelvic post-contrast CT scan demonstrating a torted ovarian cyst (C) displacing the bladder (B) anteriorly. blastoma, presacral teratoma and anterior myelomeningocoele also occur (see Table 6). most common cause. In both males and females, rhabdomyosarcoma (from prostate or vagina respectively), neuro-

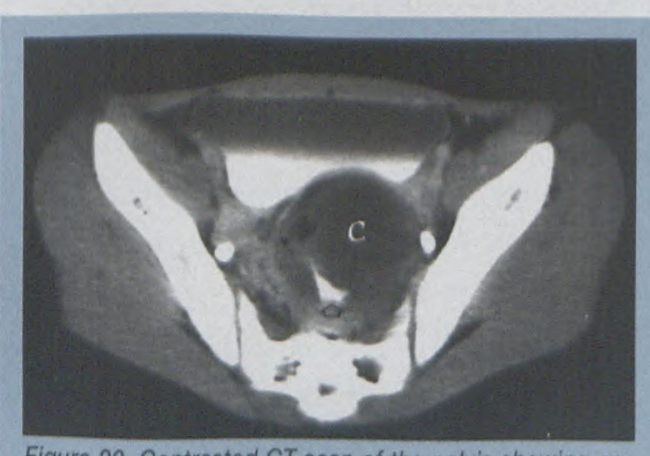

Figure 20. Contrasted CT scan of the pelvis showing an ovarian teratoma. Note the cystic component $(C)$, the calcification (arrow) and lipomatous hypodense area (arrowhead). 


\section{A review of paediatric abdominal masses}

\section{from page 20}

Cystadenocarcinoma and haematological malignancies (Figure 21) are uncommon.

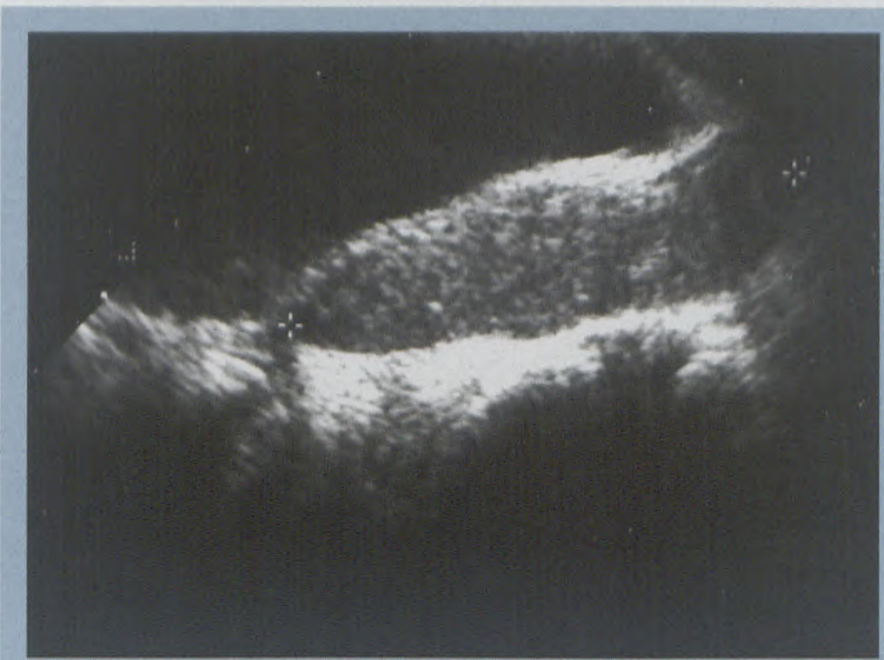

Figure 21. Pelvic ultrasound of a large left ovary (cursors) owing to leukaemic infiltration

- Haemotometrocolpos (Figure 22).

Due to an imperforate hymen or midvaginal diaphragm, this condition presents in neonates or at puberty

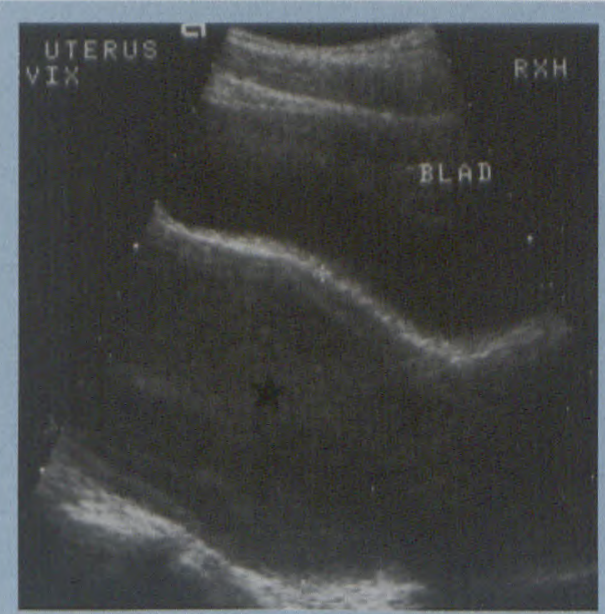

Figure 22: Sagittal ultrasound shows a large haematometrocolpos (asterisk) posterior to the bladder

with a midline lower abdominal mass arising from the pelvis. It is best appreciated on US or CT.

- Teratoma. This is the most common congenital tumour and represents approximately $40 \%$ of solid tumours in this age group. Some $60 \%$ show plain film (shard-like) calcification.
Sacrococcygeal teratomas (Figure 23) are the most common subgroup, followed by ovarian teratomas.

Retroperitoneal and gastric types are rarer., ${ }^{3,7}$

- Rhabdomyosarcoma (Figure 24) develops from primitive mesenchymal cells. It is most commonly pelvic and genitourinary in origin, especially in the bladder.

- Other causes, in no specific order of incidence,

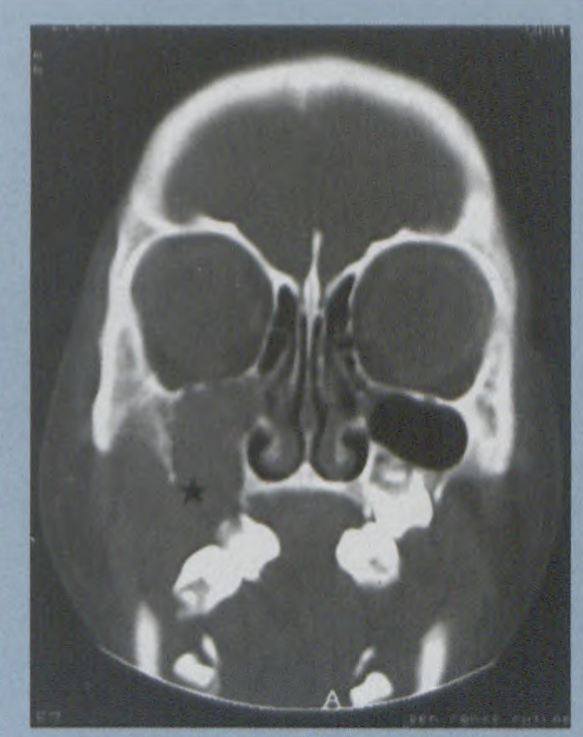

Figure 25: Coronal CT of the skull showing a soft tissue mass filling the maxillary antrum (asterisk) and eroding into the infra-temporal fossa in a child with Burkitt's lymphoma

include urachal cysts, pancreatic masses (rare, but they include cysts, gastric duplication and hamartomas), other retroperitoneal masses (apart from neuroblastoma, include lymphoma, sarcoma, teratoma and lipoma) and adrenal haemorrhage (in the acute stage may mimic neuroblastoma on US, but Figure 23: Contrasted pelvic CT scan of a sacrococcygeal teratoma (arrowheads). Note the peripheral enhancement and shard-like calcification.

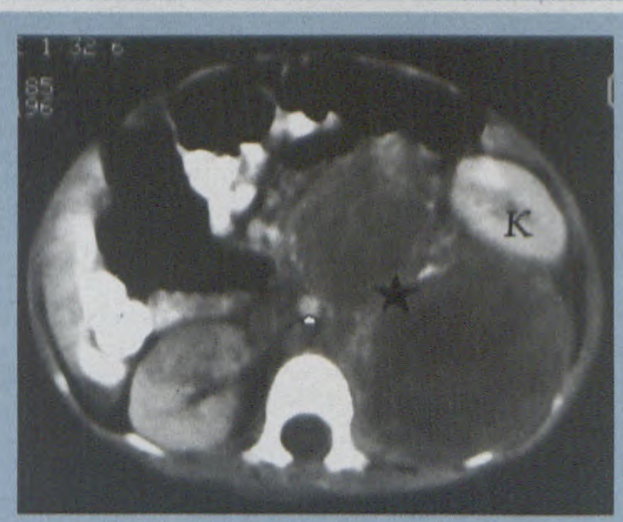

Figure 24: Contrasted abdominal CT demonstrating a heterogeneous, poorly-enhancing left retroperitoneal mass (asterisk) displacing the kidney $(K)$ anteriorly. Note the displacement of the aorta (arrow) to the right. This proved to be a rhabdomyosarcoma. will be observed to resolve on serial scans $-70 \%$ occur on the right and $5-10 \%$ are bilateral ${ }^{3}$ ). Normal and normal variant causes for an abdominal mass include an ectopic kidney, a horseshoe kidney and a distended bladder. Burkitt's lymphoma deserves a special mention in the African setting, where an abdominal mass in a patient with a jaw mass is strongly suggestive of this diagnosis (Figure 25). 